\title{
Avaliação da criminalidade no Estado do Pará
}

\author{
Assessment of crime in the State of Pará \\ Evaluación de la criminalidad en el Estado de Pará
}

Recebido: 18/02/2021 | Revisado: 26/02/2021 | Aceito: 28/02/2021 | Publicado: 08/03/2021

\author{
Hugo Alexandre Santos Regateiro \\ ORCID: https://orcid.org/0000-0003-0492-2785 \\ Universidade Federal do Pará, Brasil \\ E-mail: hregateiro@gmail.com \\ Edson Marcos Leal Soares Ramos \\ ORCID: https://orcid.org/0000-0001-5425-8531 \\ Universidade Federal do Pará, Brasil \\ E-mail: ramosedson@gmail.com \\ Joyce Gama Souza \\ ORCID: https://orcid.org/0000-0002-7852-1100 \\ Universidade Federal do Pará, Brasil \\ E-mail: joycegama7060@gmail.com \\ Cesar Maurício de Abreu Mello \\ ORCID: https://orcid.org/0000-0003-3086-2624 \\ Universidade Federal do Pará, Brasil \\ E-mail: mello.cesar@gmail.com
}

\begin{abstract}
Resumo
A violência e a criminalidade não são fenômenos presentes apenas nas grandes cidades e regiões metropolitanas, mas em todas as áreas que apresentam precarização nas condições sociais, necessitando, portanto, a compreensão das características e os impactos da criminalidade e violência nas cidades brasileiras. O objetivo do estudo é avaliar a criminalidade no Estado do Pará, Brasil, nos anos de 2017 a 2019. É um estudo quantitativo exploratório realizado nos 144 municípios do Estado do Pará, Brasil. Os dados dos crimes: (i) furto; (ii) roubo; (iii) roubo de veículo; (iv) homicídio; (v) latrocínio e (vi) lesão corporal seguida de morte, ocorridos no Pará, Brasil, foram extraídos dos Boletins de Ocorrência Policial. Os dados populacionais foram obtidos por meio do Instituto Brasileiro de Geografia e Estatística. Para mensurar a criminalidade violenta dos municípios do Pará, Brasil, utilizou-se o Índice de Criminalidade Média Bayesiana Duplamente Ponderada Padronizada para Municípios e para a apresentação espacial dos resultados, utilizaram-se os Sistemas de Informação Geográficas. Verificou-se que a maioria dos municípios apresentou índices baixos ou muito baixos de criminalidade no ano de 2019. Verificou-se, ainda, que os 5 primeiros colocados no ranking de 2018 e 2019 apresentaram índices muito alto de criminalidade. No entanto, em 2019, apenas um município apresentou índice muito alto. Foi possível avaliar a distribuição da criminalidade nos municípios do Pará, Brasil, alcançando, portanto, o objetivo do estudo. Espera-se que os resultados possam auxiliar os órgãos de segurança pública do estado na elaboração de ações efetivas nos municípios mais afetados pela criminalidade.
\end{abstract}

Palavras-chave: Violência; Crimes Violentos; Segurança Pública.

\begin{abstract}
Violence and criminality are not only present in large cities and metropolitan regions, but in all areas that are precarious in social conditions, thus requiring an understanding of the characteristics and impacts of crime and violence in Brazilian cities. The objective of the study is to assess crime in the State of Pará, Brazil, in the years 2017 to 2019. It is a quantitative exploratory study carried out in the 144 municipalities in the State of Pará, Brazil. The crime data: (i) theft; (ii) robbery; (iii) vehicle robbery; (iv) homicide; (v) robbery-homicide and (vi) bodily injury followed by death, which occurred in Pará, Brazil, were extracted from Police Reports. Population data were obtained through the Brazilian Institute of Geography and Statistics. To measure violent crime in the municipalities of Pará, Brazil, the Standardized Double-Weighted Bayesian Average Crime Index for Municipalities was used, and for the spatial presentation of the results, Geographic Information Systems were used. It was found that most municipalities had low or very low crime rates in 2019. It was also found that the top 5 placed in the 2018 and 2019 ranking had very high crime rates. However, in 2019, only one municipality had a very high rate. It was possible to assess the distribution of crime in the municipalities of Pará, Brazil, thus achieving the objective of the study. It is hoped that the results will assist the public security organs of the state in the elaboration of effective actions in the municipalities most affected by crime.
\end{abstract}

Keywords: Violence; Violent Crimes; Public security. 


\begin{abstract}
Resumen
La violencia y la criminalidad no solo están presentes en las grandes ciudades y regiones metropolitanas, sino en todas las áreas que son precarias en condiciones sociales, por lo que requieren una comprensión de las características e impactos de la delincuencia y la violencia en las ciudades brasileñas. El objetivo del estudio es evaluar la criminalidad en el estado de Pará, Brasil, en los años 2017 a 2019. Se trata de um estudio exploratorio cuantitativo realizado en los 144 municipios del estado de Pará, Brasil. Los datos del delito: (i) hurto; (ii) robo; (iii) robo de vehículos; (iv) homicidio; (v) robo seguido de muerte y (vi) lesiones corporales seguidas de muerte, ocurridos em Pará, Brasil, fueron extraídos de los informes policiales. Los datos de población se obtuvieron a través del Instituto Brasileño de Geografía y Estadística. Para medir el crimen violento en los municipios de Pará, Brasil, se utilizó el Índice de Crimen Promedio Bayesiano Doble Ponderado Estandarizado para Municipios y para la presentación espacial de los resultados se utilizaron Sistemas de Información Geográfica. Se encontró que la mayoría de los municipios tenían tasas de criminalidade bajas o muy bajas en 2019. También se encontró que los 5 primeros puestos en el ranking de 2018 y 2019 tenían tasas de criminalidad muy altas. Sin embargo, en 2019, solo un municipio tuvo una tasa muy alta. Se pudo evaluar la distribución de la delincuencia en los municipios de Pará, Brasil, logrando así el objetivo del estudio. Se espera que los resultados ayuden a las agencias de seguridad pública del estado en la elaboración de acciones efectivas en los municipios más afectados por la delincuencia.
\end{abstract}

Palabras clave: Violencia; Crímenes violentos; Seguridad Pública.

\title{
1. Introdução
}

A violência e a criminalidade são cada vez mais observadas no território brasileiro, conferindo à dinâmica social, diversas mudanças (Santos, 2016). Dessa forma, diferentemente do que se pensava, a violência e a criminalidade não são fenômenos exclusivos dos grandes centros urbanos e regiões metropolitanas, mas de qualquer território que passe pela precarização das condições sociais de vida, além do crescimento urbano desordenado (Chagas, 2014). Couto (2014) infere que a criminalidade se apresenta de várias formas, dentre elas roubos, furtos, sequestro, tráfico de drogas e homicídios.

Segundo a Organização Mundial da Saúde (OMS, 2002), as principais causas de morte para pessoas de 15 e 44 anos está ligada a violência (um fator que está diretamente relacionado a criminalidade), afirmando que trata-se de um fenômeno global caracterizado por influências específicas em diferentes regiões do mundo. A OMS (2002) considera uma situação de epidemia quando as taxas dos crimes de homicídio, por exemplo, são superiores a 10 homicídios para cada 100 mil habitantes.

No Brasil, os dados oficiais do Sistema de Informações sobre Mortalidade (SIM), do Ministério da Saúde (MS) de 2017, indicam que houve 65.602 homicídios; o que corresponde a uma taxa de aproximadamente 31,6 mortes para cada cem mil habitantes. Comparado ao ano de 2015, onde essa taxa ficou entre 55 a 60 mil homicídios, estes dados indicam que a criminalidade tornou-se crescente no país (IPEA-FBSP, 2019). À vista disso, em relação às regiões brasileiras observou-se que de 2007 a 2017 houve redução dos homicídios nas regiões Sudeste e Centro-Oeste, estabilidade do índice na região Sul, porém, houve crescimento acentuado no Norte e no Nordeste (IPEA-FBSP, 2019). Nesse cenário, o Estado do Pará apresentou, em 2017, o número de 4.575 dos crimes de homicídios (IPEA-FBSP, 2019).

Nesse contexto, justifica-se o artigo se pela necessidade de compreender como se dá a distribuição da criminalidade no Estado do Pará, possibilitando estabelecer indicadores de criminalidade que possam colaborar com os órgãos de segurança pública do Estado do Pará, no desenvolvimento de estratégias que o objetivem minimizar a ocorrência da criminalidade no estado. Nessa perspectiva, o objetivo do estudo é compreender como se dá a distribuição da criminalidade no Estado do Pará, nos anos de 2017 a 2019.

\section{Metodologia}

Trata-se de um estudo quantitativo exploratório realizado nos 144 municípios do Estado do Pará, localizado na região Norte do país, demarcando 1.247.955,24 km² de área e uma população de aproximadamente 8.366.628 habitantes no ano de 2017 (IBGE, 2018). Os dados referentes aos Registros de Boletim de Ocorrência (B.O.P.) dos crimes (i) furto; (ii) roubo; (iii) roubo de veículo; (iv) homicídio; (v) latrocínio e (vi) lesão corporal seguida de morte, ocorridos no Estado do Pará, nos anos 
de 2017, 2018 e 2019, foram extraídos do Sistema Integrado de Segurança Pública (SISP) e disponibilizados por meio da Secretaria Adjunta de Inteligência e Análise Criminal (SIAC).

A coleta dos dados populacionais, necessários à obtenção dos indicadores de criminalidade dos municípios do Estado do Pará, foi realizada a partir das informações disponibilizadas pelo IBGE (2018). Para a utilização da Taxa de Criminalidade Média Bayesiana Ponderada Padronizada para Município (TCMBPPM) e do Índice de Criminalidade Média Bayesiana Duplamente Ponderada Padronizada para Município (ICMBDPPM) (Regateiro et al., 2021) na variável roubo de veículos, utilizou-se dados do Departamento Nacional de Trânsito referentes à frota veicular dos municípios do Estado do Pará, nos anos de 2017,2018 e 2019.

Inicialmente, utilizou-se a técnica estatística análise exploratória de dados para relacionar variáveis e compreender o fenômeno estudado e, posteriormente organizá-los visualmente utilizando-se de gráficos, tabelas e medidas de síntese (Bussab \& Moretin, 2017). Para obtenção de um indicador de criminalidade por tipologia criminal (furto, roubo, homicídio, latrocínio e lesão corporal seguida de morte) para os municípios do Estado do Pará, utilizou-se a Taxa de Criminalidade Média Bayesiana Ponderada Padronizada para Município (TCMBPPM) (Regateiro et al., 2021), cujos Escores, isto é, valores resultantes para cada crime e município, são avaliados conforme a Figura 1. Para mensurar criminalidade dos municípios do estado, utilizou-se o Índice de Criminalidade Média Bayesiana Duplamente Ponderada Padronizada para Município (ICMBDPPM) (Regateiro et al., 2021), cujos Escores são avaliados conforme Figura 1.

Figura 1 - Escala de Avaliação da Taxa de Criminalidade Média Bayesiana Ponderada Padronizada para Município e do Índice de Criminalidade Média Bayesiana Duplamente Ponderada Padronizada para Município.

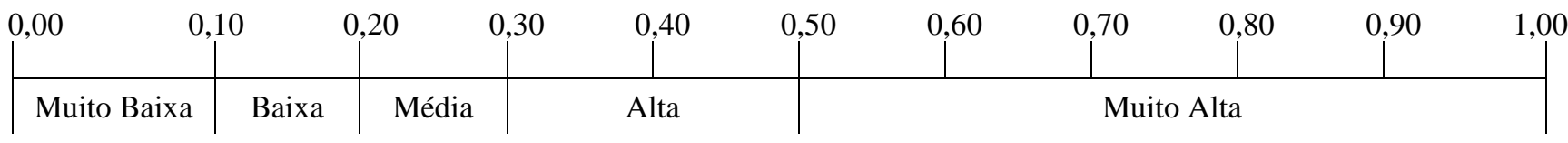

Fonte: Regateiro et al. (2021).

Para apresentação da distribuição espacial dos níveis de criminalidade dos municípios do Estado do Pará, utilizaramse ferramentas de Sistemas de Informação Geográficas (SIG) a partir do software ARCGIS Geostatistical Analyst (Câmara et al., 2002).

\section{Criminalidade, Espaço, Periferização e Territorialização}

O crime é uma manifestação humana, desenvolvido por diversos fatores (Xavier, 2008). Para entender este fenômeno, criaram-se várias teorias que buscaram esclarecer as motivações para ocorrência de delitos e quais fatores viabilizam o acontecimento do mesmo. Por ser uma característica comportamental humana motivada por inúmeras causas, não é possível fazer generalizações a respeito da criminalidade, pois não se trata de algo singular, mas está entrelaçada a dinâmica social, cultural e política, além de fatores econômicos e pessoais em que os indivíduos estão inseridos (Baltazar et al., 2011).

Segundo Procópio e Toyoshima (2017) os principais fatores que contribuem para a criminalidade no Brasil estão diretamente relacionados à desigualdade social, processo de urbanização das cidades, má distribuição dos recursos em setores básicos como educação e o crescimento de atividades relacionadas ao tráfico de drogas. Para Chagas (2014) a construção do espaço urbano, além do processo de territorialização da violência e do crime, predispõem fatores influentes à criminalidade. $\mathrm{O}$ processo de construção do espaço urbano, quando realizado de maneira desordenada, promove a segregação social, pobreza e favelização, evidenciadas nas áreas periféricas, as quais refletem o abandono e a desvalorização pelo poder público (Chagas, 2014). Dessa forma, as áreas periféricas são vistas como preponderantes à violência e criminalidade, segregando ainda mais os territórios e, consequentemente, rotuladas como áreas a serem controladas ostensivamente pelo estado (Haesbaert, 2014). 
Outro fator importante no entendimento a respeito da criminalidade é o processo de territorialização que, segundo Raffestin (2000), a ausência do estado nas regiões periféricas dos centros urbanos, cria um ambiente propício à disputa territorial pelos agentes, isto é, representantes comunitários, igrejas, pequenos empresários e grupos criminosos. Nesse contexto, locais excluídos onde há ausência do estado, potencializam o estabelecimento de atividades criminosas e zonas de tensão, as quais possuem como forma de controle a violência (Chagas, 2014).

No contexto específico do Estado do Pará, no ano de 2017, foram registrados no estado, 121.449 ocorrências de Furto, previsto no Artigo 155 do Código Penal Brasileiro como a subtração de coisa alheia móvel para si ou para outrem (Pará, 2020a). Em relação às ocorrências de roubo, isto é, subtração para si ou para outrem de coisa móvel alheia a partir de grave ameaça ou violência à pessoa, previsto no Artigo 157, o estado registrou 130.574 ocorrências (Pará, 2020b). O Artigo 121 do Código Penal tipifica que matar alguém é crime de homicídio, o qual foram registrados 3.887 homicídios no Pará em 2017 (Pará, 2020c). Para o crime latrocínio, previsto no Artigo 157, § $3^{\circ}$ (trata-se de uma qualificadora do crime de roubo, aumentando a pena quando se tem o resultado morte), houve 228 registros no Estado do Pará em 2017 (Pará, 2020d). Ainda no ano de 2017, o Pará registrou 40 ocorrências do crime lesão corporal seguida de morte, o qual está pre visto no Artigo 129, $\S 3^{\circ}$ do Código Penal Brasileiro, isto é, ofender a integridade física de alguém, tendo como resultado a morte (Pará, 2020e).

Dessa forma, a compreensão da criminalidade e suas características por parte dos órgãos da Segurança Pública, são imprescindíveis ao planejamento de ações e políticas. Contudo, de acordo com Lemgruber et al. (2002), as políticas de segurança enfrentam problemas para desenvolver estratégias que facilitem o diagnóstico preciso dos problemas em relação à criminalidade e, por conta disso as intervenções são imediatistas, assistemáticas e reativas, demonstrando a falta de planejamento e metodologias adequadas capazes de mensurar de forma objetiva a magnitude, a natureza e as circunstâncias dos crimes cometidos. Portanto, é significativa a importância de estudos que desenvolvam que descrevam o andamento e as diferenças regionais da criminalidade no país, além de identificar a eficácia das políticas públicas no combate a mesma.

\section{Resultados e Discussão}

A Figura 2 apresenta a quantidade de municípios do Pará, por nível de criminalidade, para os anos de 2017, 2018 e 2019, obtido a partir do Índice de Criminalidade Média Bayesiana Duplamente Ponderada Padronizada para Municípios (ICMBDPPM). Nela, pode-se observar que o Pará apresenta, no período estudado, a maioria dos municípios com índices baixos ou muito baixos de criminalidade (Figura 2). Em 2019, evidencia-se um aumento na quantidade de municípios com níveis baixos e muito baixos de criminalidade (Figura 2). Além disso, nota-se, ainda, em 2019, que apenas 1 município do Pará, apresenta nível muito alto de criminalidade (Figura 2). 
Figura 2 - Quantidade de Municípios do Pará, por nível de criminalidade, para os anos de 2017, 2018 e 2019, obtidos a partir do Índice de Criminalidade Média Bayesiana Duplamente Ponderada Padronizada para Município.

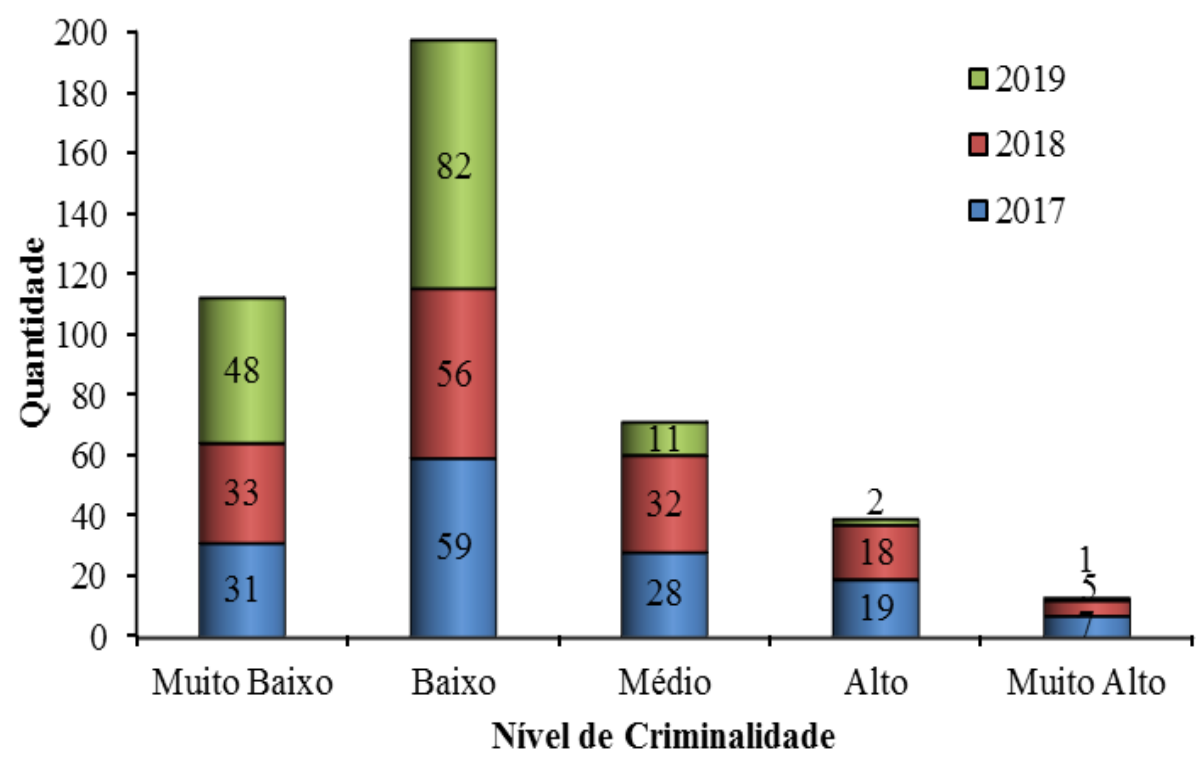

Nota: Escala ICMBDPPM: 0 a 0,10 - Muito Baixa; 0,11 a 0,20 - Baixa; 0,21 a 30 - Média; 0,31 a 0,50 - Alta; 0,51 a 1,00 Muito Alta (Regateiro et al., 2021). Fonte: Construção dos Autores.

Em contraposição a estudos que ratificam a violência como característica de grandes centros urbanos e regiões metropolitanas, Endlich e Fernandes (2014) enfatizam que a criminalidade é um fenômeno crescente em cidades pequenas e apontam como principais causas a ausência de políticas públicas de segurança, possibilitando, portanto, a interiorização da violência. As Figuras 3, 4 e 5, apresentam os mapas com a classificação da criminalidade dos 144 municípios do Pará, obtidos a partir do Índice de Criminalidade Média Bayesiana Duplamente Ponderada Padronizada por Município, para os anos de 2017,2018 e 2019.

Dessa forma, verificou-se que no ano de 2017 (Figura 3), segundo o ICMBDPPM, 7 municípios paraenses apresentaram índice de criminalidade "muito alto", dos quais, ocupando as 5 primeiras posições do ranking, destacaram-se os municípios Moju (0,6282), Igarapé-Miri (0,5973), Curionópolis (0,5882), Marituba (0,5818) e Jacareacanga (0,5799). Dentre os municípios que ocupam as primeiras posições no ranking, apenas o Município de Marituba integra a região Metropolitana de Belém (Figura 3). 
Figura 3 - Criminalidade nos municípios do Estado do Pará no período de Janeiro a Dezembro de 2017.

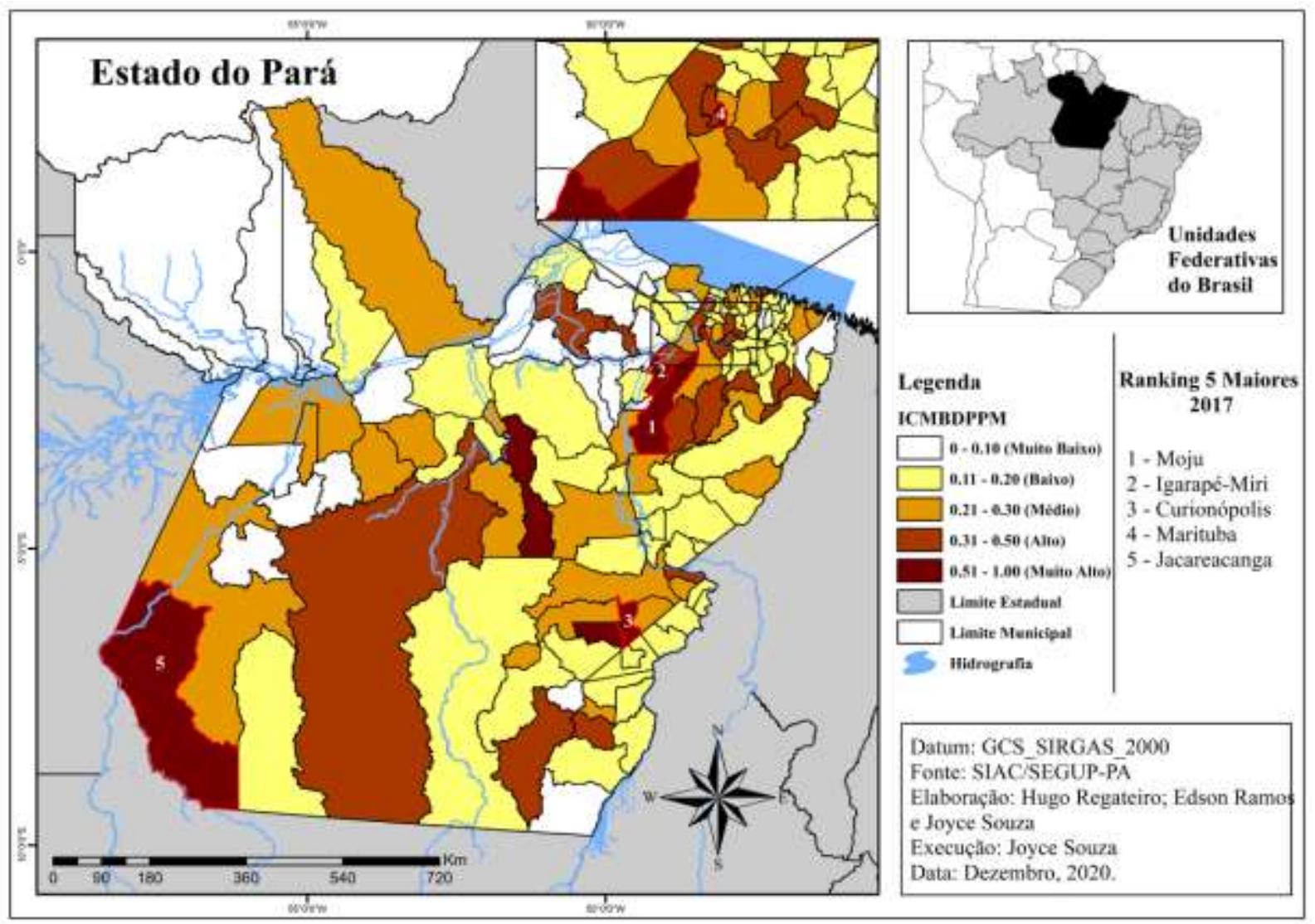

Fonte: Construção dos Autores.

O município Moju, localizado na região leste do estado, demarca uma área de $9.094,139 \mathrm{~km}^{2}$ e detém uma população de 83.182 habitantes (IBGE, 2020a). Além disso, no ano de 2010, o município apresentou somente 5,5\% do seu território com esgotamento sanitário adequado, apenas 2,4\% das vias públicas urbanizadas, 9,7\% da população ocupada, isto é, trabalhando e IDHM de 0,547 (IBGE, 2020a).

O município Igarapé-Miri, localiza-se na região leste do Estado do Pará demarcando uma área de $1.996,790$ km² e apresenta uma população de 58.077 habitantes (IBGE, 2020b). No ano de 2010, não havia urbanização de vias públicas no município, apenas 3,3\% do seu território possuía esgotamento sanitário adequado, apenas 6,3\% dos habitantes ocupados para o trabalho e IDHM de 0,547 (IBGE, 2020b).

O município Curionópolis, localizado ao sul do Estado do Pará, demarca uma área de 2.369,098km² e uma população de 17.846 habitantes (IBGE, 2020c). Em 2010, o município apresentou apenas 0,2\% de vias públicas urbanizadas, 5,2\% de esgotamento sanitário adequado, 14,7\% da população ocupada para o trabalho e IDHM de 0,636 (IBGE, 2020c).

O município Marituba, localizado a $11 \mathrm{~km}$ de distância da capital Belém, integrando a Região Metropolitana de Belém, demarca uma área de 103.214 km² e uma população de 133.685 habitantes, tornando-se um dos municípios mais populosos do Estado do Pará (IBGE, 2020d). No ano de 2010, o município Marituba apresentou somente 0,4\% de suas vias públicas urbanizadas, $18,8 \%$ de esgotamento sanitário adequado, 13,8\% da sua população ocupada para o trabalho e IDHM de 0,676 (IBGE, 2020d).

O município Jacareacanga, localiza-se ao sul do Estado do Pará, demarca uma área de 53.304,564 km² e uma população de 7.590 habitantes (IBGE, 2020e). No ano de 2010, Jacareacanga apresentou 4,7\% das vias públicas urbanizadas, apenas $2 \%$ de esgotamento sanitário adequado, 17,9\% da população ocupada para o trabalho e IDHM de 0,505 (IBGE, 2020e). 
Além disso, no ano de 2017, 59 municípios do estado apresentaram "baixos" índices de criminalidade e 31 municípios apresentaram índices "muito baixos", dos quais o município de Bannach apresentou o menor índice $(0,0668)$ (Figura 3).

Figura 4 - Criminalidade nos municípios do Estado do Pará no período de Janeiro a Dezembro de 2018.

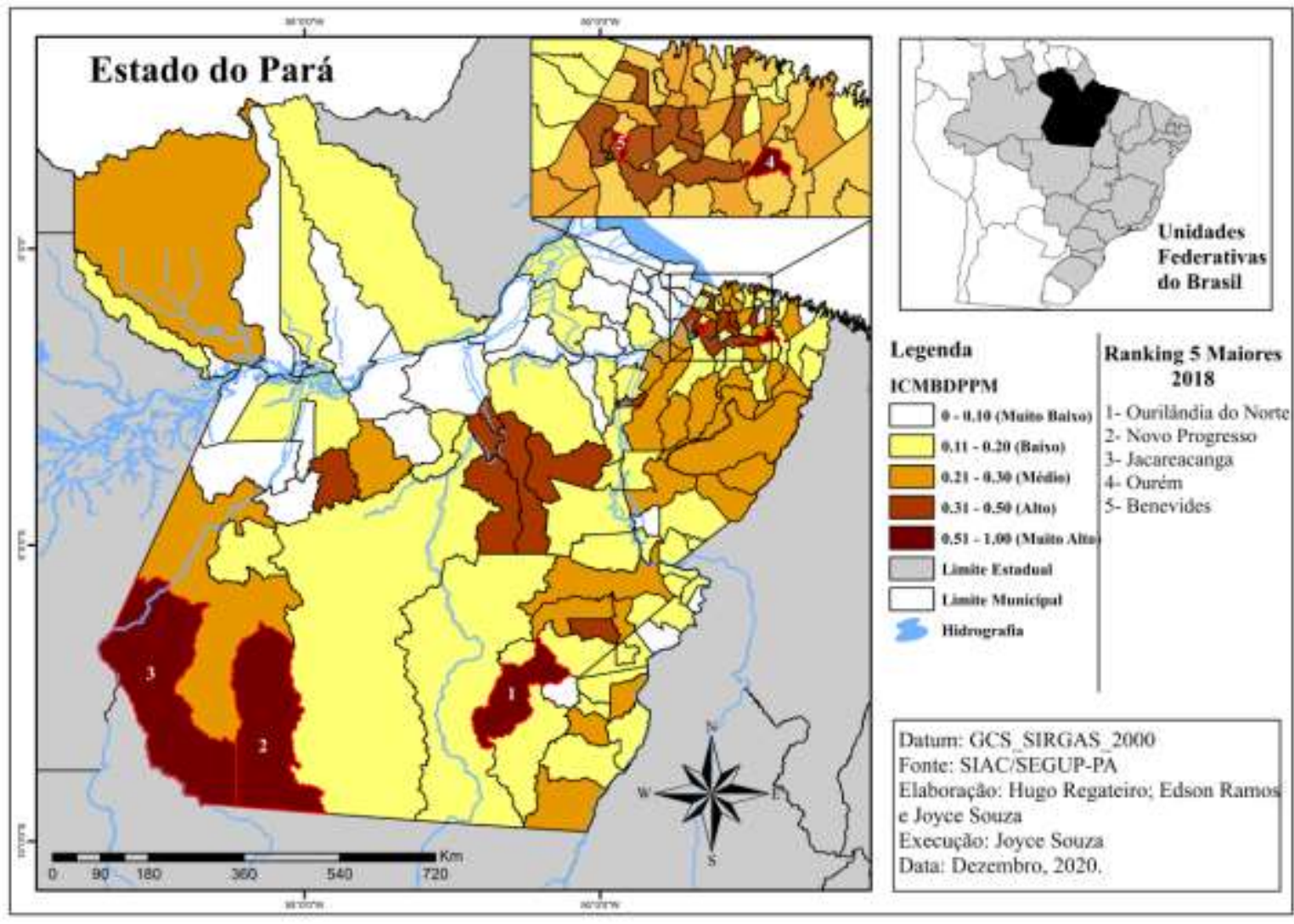

Fonte: Construção dos Autores.

No ano de 2018 os municípios Ourilândia do Norte (0,6310), Novo Progresso $(0,6128)$, Jacareacanga $(0,5995)$, Ourém $(0,5416)$ e Benevides $(0,5082)$ apresentaram os maiores índices de criminalidade no Estado do Pará e foram classificados no nível de criminalidade "muito alto" (Figura 4). Ainda na Figura 4, observa-se que dentre os 5 primeiros colocados no ranking, apenas o Município Benevides (5 colocado) integra a Região Metropolitana de Belém. Dos 144 municípios do estado, 56 municípios apresentaram índices "baixos" e 33 "muito baixos". Em relação aos municípios que apresentaram os menores índices, destacam-se os municípios de Bannach (0,0688), Curuá (0,0626) e Chaves $(0,0596)$ (Figura 4).

O município Ourilândia do Norte localiza-se ao sul do Estado do Pará, demarca uma área de 14.410,567 km² e uma população de 33.335 habitantes (IBGE, 2020f). No ano de 2010, o município apresentou apenas 0,5\% das vias públicas urbanizadas, apenas 8,7\% de esgotamento sanitário adequado, 17,9\% da população ocupada para o trabalho e IDHM de 0,624 (IBGE, 2020f).

O município Novo Progresso, localizado ao sul do Estado do Pará, demarca uma área de 38.162,013 km² e uma população de 25.766 habitantes (IBGE, 2020g). No ano de 2010, o município apresentou apenas 1,4\% de esgotamento sanitário adequado, apenas $0,1 \%$ das vias públicas urbanizadas, $18,2 \%$ da população ocupada para o trabalho e IDHM de 0,673 (IBGE, 2020g).

O município Ourém, localizado ao sul do Estado do Pará, demarca uma área de 561,710 km² e uma população de 17.961 habitantes (IBGE, 2020h). No ano de 2010, o município apresentou apenas 12,7\% de esgotamento sanitário adequado, 6,1\% das vias públicas urbanizadas, $5,3 \%$ da população ocupada para o trabalho e IDHM de 0,568 (IBGE, 2020h). 
O município Benevides, localiza-se a $25 \mathrm{~km}$ de distância da capital Belém, integrando a Região Metropolitana de Belém, possui uma população de 51.651 habitantes e demarca uma área de 187,826 km² (IBGE, 2020i). Em 2010, o município apresentou apenas $0,9 \%$ das vias públicas urbanizadas, apenas 17,4\% de esgotamento sanitário adequado, 16,7\% da população ocupada para o trabalho e IDHM de 0,665 (IBGE, 2020i).

Na Figura 5, observa-se que no ano de 2019, apenas município de Bannach $(0,6050)$, apresentou índice de criminalidade "muito alto", passando das últimas posições do ranking nos anos de 2017 e 2018, para a primeira posição do ranking no ano de 2019.

O município Bannach, localizado ao sul do Estado do Pará, demarca uma área de 2.956,649 km² e uma população de 3.262 habitantes (IBGE, 2020j). No ano de 2010, o município apresentou apenas 1,8\% de esgotamento sanitário adequado, nenhuma via pública urbanizada, 12,6\% da população ocupada para o trabalho e IDHM de 0,594 (IBGE, 2020j).

Dos 144 municípios do estado, 82 municípios apresentaram "baixo" índice de criminalidade e 48 municípios do Estado do Pará apresentaram um índice de criminalidade "muito baixo". Dos municípios que apresentaram índices "muito baixos", destacam-se Limoeiro do Ajuru $(0,0555)$, Cachoeira do Arari $(0,0528)$ e Chaves $(0,518)$ ocupando consecutivamente, ou seja, nos anos de 2018 e 2019, a última posição no ranking (Figura 5).

Segundo Weyrauch (2011) a prática da violência, a qual está diretamente ligada aos índices de criminalidade, consolida-se de várias formas, individuais ou coletivas, a partir da ausência de direitos sociais os quais influenciam diretamente no campo público ou privado, nos meios de produção e, sobretudo, no processo de urbanização das cidades. Dessa forma, as características urbanísticas, sociopolíticas, culturais, além do número de habitantes, bem como, a consciência comunitária dos mesmos, são características históricas da violência e, consequentemente, contribuem para o aumento da criminalidade (Weyrauch, 2011).

Figura 5 - Criminalidade nos municípios do Estado do Pará no período de Janeiro a Dezembro de 2019.

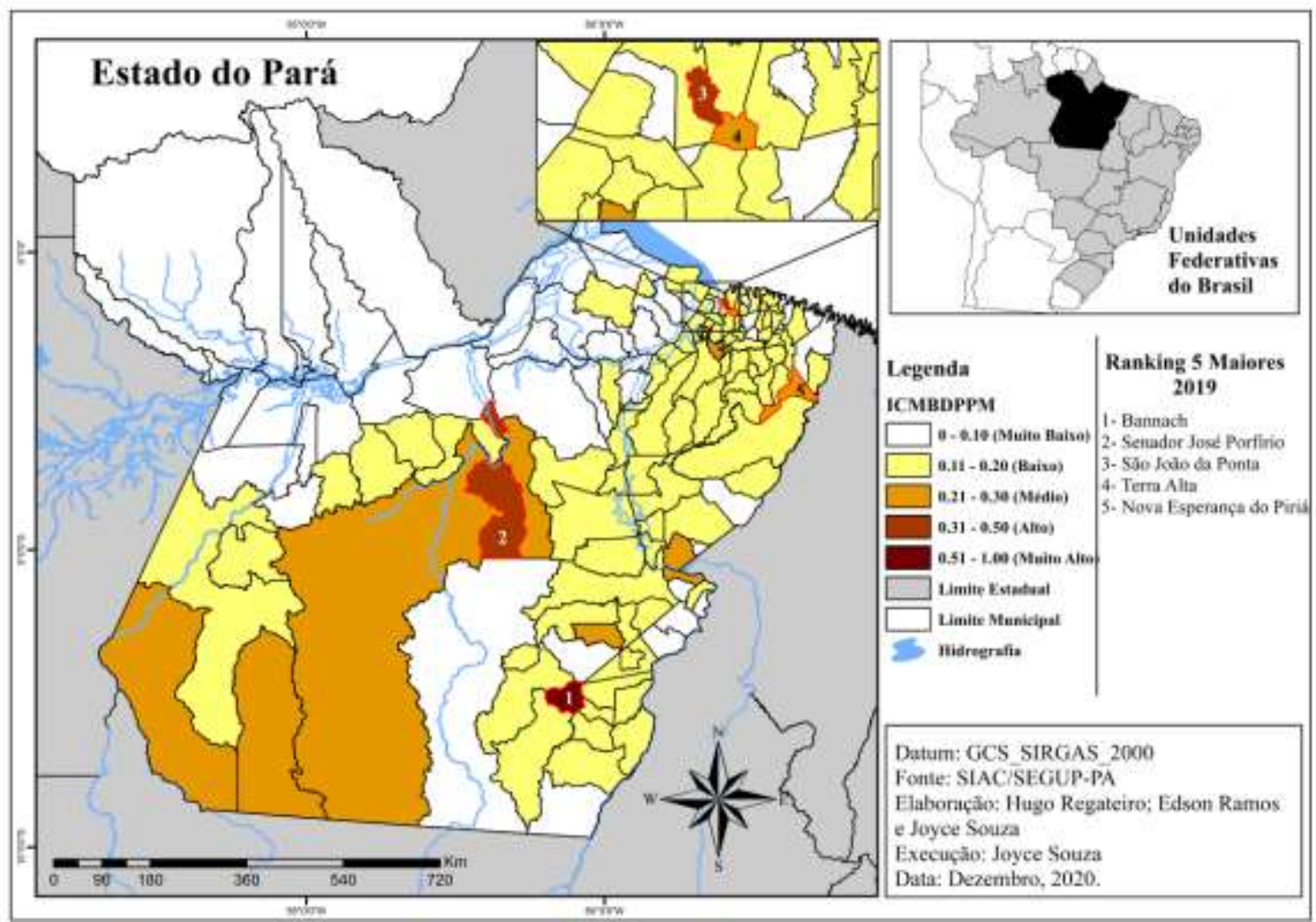

Fonte: Construção dos Autores. 
Para Garrido (2016) os sistemas capitalista e político estão diretamente relacionados ao processo de criminalidade, apontando a má distribuição de riquezas, as crises econômicas e a carência de programas assistenciais e governamentais como fatores que corroboram à criminalidade. Outra afirmativa desenvolvida por, Garrido (2016, p. 5) destaca que políticas eficazes de enfrentamento à criminalidade perpassam pela "proteção à infância, a habitação, o regime de aposentadoria, a política do salário, a saúde" entre outros.

Ainda no campo da criminalidade, Gomes (2016) destaca as diferenças dentre os índices de criminalidade, ratificando que os países com os maiores índices apresentam algumas características como desigualdades sociais, econômicas, baixos índices de escolaridade, baixos IDHM's e altos índices de corrupção, enquanto que os países menos violentos apresentam características inversas, isto é, apresentam IDHM's elevados ou muito elevados, ótimos índices de escolaridade, baixos índices de corrupção e desigualdades econômicas e sociais. Nesse contexto, verificou-se que, dos 10 municípios do Estado do Pará que permaneceram dentre os 5 maiores no ranking, apresentando índices muito alto de criminalidade no período de 2017 a 2019, os municípios Marituba, Benevides, Ourilândia do Norte, Curionópolis e Novo Progresso apresentaram IDHM médio, dos quais 2 municípios integram a Região Metropolitana de Belém enquanto os municípios Moju, Igarapé-Miri, Jacareacanga, Ourém e Bannach apresentaram IDHM baixos.

Um estudo realizado por Waiselfisz (2010) sobre a ocorrência de homicídios nas cidades do Brasil, apontou que 9 das 30 cidades, que ocuparam as primeiras posições do ranking de homicídios, estimavam uma população inferior a 20.000 habitantes, concluindo, assim, que a criminalidade independe da cidade e suas características como o tamanho populacional, por exemplo. Dessa forma, observou-se que dos 10 municípios que apresentaram os maiores índices de criminalidade nos últimos três anos, ocupando as primeiras 5 posições no ranking, os municípios Moju, Igarapé-Miri, Curionópolis, Jacareacanga, Ourilândia do Norte, Novo Progresso, Ourém, Benevides e Bannach são consideradas cidades de pequeno porte, detendo uma população menor que 100.000 habitantes e apenas o município Marituba apresenta uma população de 133.685 habitantes.

Gomes (2016) destaca que o investimento em serviços públicos de educação, saúde, segurança pública e saneamento básico são imprescindíveis à elevação da qualidade de vida e consequentemente, no IDHM como possível medida para redução nos índices de criminalidade no Brasil. Entretanto, Tavares (2016), infere que, apesar do Brasil apresentar recordes de recolhimento tributário, a devolução dos impostos em serviços básicos é escassa, implicando em sérios problemas na educação, saúde, segurança e saneamento básico, tornando o ambiente propício à criminalidade. Nesse contexto, verificou-se que os municípios do Estado do Pará que apresentaram os maiores índices de criminalidade, permanecendo nas primeiras posições do ranking no período de 2017 a 2019, apresentaram péssimos níveis de esgotamento sanitário adequado e vias públicas urbanizadas, sendo estas, inexistentes no município de Bannach.

Outra característica importante no entendimento da criminalidade é a taxa de desemprego a qual, Carvalho et al. (2017) destacam que esta tem se elevado nos últimos anos no Brasil devido as condições econômicas que o país enfrenta. Nesse cenário, Araújo e Antigo (2015) destacam que a taxa de desocupação implica no aumento da pobreza e, consequentemente, no aumento da criminalidade visto que, o desempregado encontra nas ações ilícitas, independente do sucesso ou não na execução da mesma, fonte de sobrevivência. Dessa forma, um estudo realizado na cidade de Santarém, no Estado do Pará, apontou que a crescente criminalidade na região está associada ao desemprego, destacando a insatisfação dos moradores locais com a falta de empregos, colaborando para a ocorrência de roubos e furtos (Carvalho et al., 2017). No contexto específico dos municípios que apresentaram índices muito altos de criminalidade, verificou-se que os municípios Curionópolis, Marituba, Jacareacanga, Ourilândia do Norte, Novo Progresso e Benevides apresentaram uma taxa de ocupação para o trabalho acima de 10\% para a sua população enquanto os municípios de Moju, Igarapé-Miri e Ourém apresentaram uma taxa de ocupação para o trabalho menor que $10 \%$. 


\section{Conclusão}

Verificou-se no estudo, o qual se objetivou avaliar a distribuição da criminalidade no Estado do Pará nos anos de 2017, 2018 e 2019, que houve redução nos índices de criminalidade na maioria dos municípios, no período estudado, o qual se verificou que no ano de 2019 a maioria dos municípios apresentou índices baixo ou muito baixo de criminalidade. Entretanto, verificou-se, ainda, que os 5 primeiros colocados no ranking apresentaram índices muito altos de criminalidade nos anos de 2017 e 2018. Entretanto, no ano de 2019, apenas o município de Bannach apresentou índice muito alto de criminalidade.

Dos municípios destacados no ranking com maiores índices de criminalidade, no período de 2017 a 2019, verificou-se que todos apresentaram algumas características que podem estar associadas à criminalidade, dentre elas, as péssimas condições de saneamento básico, urbanização, baixos IDHM's e taxa de ocupação para o trabalho.

Diante do exposto, identificaram-se as distribuições geográficas da criminalidade nos municípios do estado Pará. A partir dos resultados encontrados, espera-se que os mesmos sejam utilizados como apoio aos gestores dos sistemas de segurança pública do estado no desenvolvimento das ações de combate à criminalidade nos municípios mais violentos abordados no estudo, bem como, no estado. Espera-se, ainda, que este estudo forneça subsídios na busca de novas metodologias que possam trabalhar de forma eficiente os dados referentes à segurança pública.

\section{Referências}

Araújo, J. P. F., \& Antigo, M. F. (2015). Desemprego e qualificação da mão de obra no Brasil. Revista de Economia Contemporânea, 20 (2), $308-335$.

Baltazar, C. S., Stocki, J. F., \& Kafrouni, R. (2011). O conceito de Crime e Criminalidade para agentes de segurança da cidade de Curitiba. Revista Polis e Psique, 1 (1), 110-129.

Bussab, W. O., \& Morettin, P. A. (2017). Estatística Básica. (9a ed.): Saraiva.

Câmara, G., Monteiro, A. M., Fucks, S. D., \& Carvalho, M. S. (2002). Análise espacial de dados geográficos. (1a ed.): INPE.

Carvalho, B. N., Lobato, T. C., De Carvalho, A. V., \& Guimarães, J. L. C. (2017). Relação da Criminalidade com os Índices de Desemprego: um estudo de caso. Enciclopédia Biosfera, Centro Científico Conhecer - Goiânia, 14 (25), 1804-1817.

Chagas, A. C. N. (2014). Geografia, segurança pública e a cartografia dos homicídios na Região Metropolitana de Belém. Revista boletim amazônico da Geografia, 1 (1), 186-204.

Couto, A. C. O. (2014). A geografia do crime: das redes ilegais à "territorialização perversa" na periferia de Belém. EDUEPA.

Endlich, A. M., \& Fernandes, P. H. C. (2014). Aumento da violência em pequenas cidades, sentimento de insegurança e controle social. In: XIII Coloquio Internacional de Geocrítica, (pp. 5-10). Barcelona: Universidade de Barcelona.

Garrido, A. C. O. (2016). Fatores Sociais De Criminalidade. http://www.atenas.edu.br/uniatenas/assets/files/magazines/FATO RES_SOCIAIS_DE_CRIMINALIDADE_.pdf.

Gomes, L. F. (2016). O Mito da Segurança Grátis - Brasil: 12º mais violento do planeta. http://institutoavantebrasil.com.br/o-mito-da-seguranca-gratis-brasil12o-mais-violento-do-planeta/.

Haesbaert, R. (2014). Viver no limite: território e multi/ transterritorialidade em tempos de in-segurança e contenção. Bertrand Brasil.

Instituto Brasileiro de Geografia e Estatística. (2018). Cidades. https://cidades.ibge.gov.br/brasil/pa/panorama.

Instituto Brasileiro de Geografia e Estatística. (2020a). Cidades. https://cidades.ibge.gov.br/brasil/pa/moju/panorama.

Instituto Brasileiro de Geografia e Estatística. (2020b). Cidades. https://cidades.ibge.gov.br/brasil/pa/igarape-miri/panorama.

Instituto Brasileiro de Geografia e Estatística. (2020c). Cidades. https://cidades.ibge.gov.br/brasil/pa/curionopolis/panorama.

Instituto Brasileiro de Geografia e Estatística. (2020d). Cidades. https://cidades.ibge.gov.br/brasil/pa/marituba/panorama.

Instituto Brasileiro de Geografia e Estatística. (2020e). Cidades. https://cidades.ibge.gov.br/brasil/pa/jacareacanga/panorama.

Instituto Brasileiro de Geografia e Estatística. (2020f). Cidades. https://cidades.ibge.gov.br/brasil/pa/ourilandia-do-norte/panorama.

Instituto Brasileiro de Geografia e Estatística. (2020g). Cidades. https://cidades.ibge.gov.br/brasil/pa/novo-progresso/panorama.

Instituto Brasileiro de Geografia e Estatística (2020h). Cidades. https://cidades.ibge.gov.br/brasil/pa/ourem/panorama. 
Research, Society and Development, v. 10, n. 3, e10010313088, 2021

(CC BY 4.0) | ISSN 2525-3409 | DOI: http://dx.doi.org/10.33448/rsd-v10i3.13088

Instituto Brasileiro de Geografia e Estatística. (2020i). Cidades. https://cidades.ibge.gov.br/brasil/pa/benevides/panorama.

Instituto Brasileiro de Geografia e Estatística. (2020j). Cidades. https://cidades.ibge.gov.br/brasil/pa/bannach/panorama.

Instituto de Pesquisa Econômica Aplicada e Fórum Brasileiro de Segurança Pública. (2019). Atlas da Violência, 6-24.

Lemgruber, J., Musumeci, L., \& Ramos, S. (2002). Por que é tão difícil implementar uma política de segurança? Observatório da Cidadania, p. 46.

Organização Mundial da Saúde. (2002). World report on violence and health. https://www.who.int/violence injury_prevention/violence/world_report/en/full_en.pdf?ua=1.

Pará. Secretaria de Segurança Pública e Defesa Social. (2020a). Furtos 2017. http://sistemas.segup.pa.gov.br/transparencia/roubo-2017/.

Pará. Secretaria de Segurança Pública e Defesa Social. (2020b). Roubos 2017. http://sistemas.segup.pa.gov.br/transparencia/furtos-2017/.

Pará. Secretaria de Segurança Pública e Defesa Social. (2020c). Homicídios 2017. http://sistemas.segup.pa.gov.br/transparencia/homicidios-2017/.

Pará. Secretaria de Segurança Pública e Defesa Social. (2020d). Latrocínios 2017. http://sistemas.segup.pa.gov.br/transparencia/latrocinios-2017/.

Pará. Secretaria de Segurança Pública e Defesa Social. (2020e). Lesão Corporal Seguida de Morte 2017. http://sistemas.segup.pa.gov.br/transparencia/lcsm$2017 /$.

Procópio, D. P., \& Toyoshima, S. H. (2017). Fatores Associados à Criminalidade Violenta no Brasil. Análise Econômica, 35, 263-288.

Raffestin, C. (2000). Por uma geografia do Poder. Ática.

Regateiro, H. A. S., Ramos, E. M. L. S., \& Souza, J. G. (2021). Índice de Criminalidade Média Bayesiana Duplamente Ponderada Padronizada para Municípios. http://educapes.capes.gov.br/handle/capes/585837.

Santos, M. A. F. (2016). Abordagens científicas sobre as causas da criminalidade violenta: uma análise da teoria da ecologia humana. Revista do Laboratório de Estudos da Violência da UNESP/Marília, (17), p. 46-77.

Tavares, E. A. (2016). O Aumento da Criminalidade no Brasil: uma relação direta com o IDH Brasileiro. Revista SynThesis, 7 (7), 229-239.

Xavier, A. (2008). A construção do conceito de criminoso na sociedade capitalista: um debate para o Serviço Social. Revista Katálysis, 11(2), $274-282$.

Waiselfisz, J. J. (2010). Mapa da violência 2010: anatomia dos homicídios. OEI.

Weyrauch, C. S. (2011). Violência urbana. Dimensões, 27, 2-22. 